\title{
OUTAGE ANALYSIS OF LOW-LATENCY COOPERATIVE WIRELESS NETWORKS WITH THRESHOLD-BASED PROTOCOL OVER COMPOSITE FADING
}

\author{
$U D C(621.391+621.395 .38): 519.724$
}

\begin{abstract}
Nemanja Zdravković
University of Niš, Faculty of Electronic Engineering, Department of Telecommunications, Niš, Republic of Serbia
\end{abstract}

\begin{abstract}
This paper studies a low-latency decode-and-forward cooperative wireless network subject to composite fading. Assuming temporally correlated channel between cooperating nodes and maximal ratio combining at the destination, outage probability $(O P)$ performance is investigated and novel OP expressions are derived when nodes apply a threshold-based protocol for internode communication. The effects of network dimension, multipath fading and shadowing severity parameters, correlation coefficients and average uplink and internode signal-to-noise ratios on $O P$ are discussed.
\end{abstract}

Key words: composite fading, cooperative communication, decode-and-forward relay, shadowing, outage probability.

\section{INTRODUCTION}

Wireless communication systems continue to evolve, keeping up with the constantly growing user demands for better coverage, higher data rates and overall seamless user experience. Utilizing cooperative diversity full spatial diversity can be achieved and therefore overall network performance can be improved [1]. Cooperative protocols can be divided into two main protocols and their derivations - amplify-and-forward (AF) and decode-and-forward [2]. The simplest protocol is AF, where the signal received by a relay node is amplified, frequency translated and retransmitted. Amplification can be performed by either a fixed or variable gain, which require channel state information (SCI) [3,4]. In DF protocols, a relay node detects the signal, decodes it, re-encodes and only then retransmits it. These protocols require more hardware and are more complex in processing terms compared to $\mathrm{AF}$, however, $\mathrm{DF}$ protocols are known to be a performance optimum w.r.t. metrics such as

Received December 13, 2016

Corresponding author: Nemanja Zdravković

University of Niš, Faculty of Electronic Engineering, Department of Telecommunications, Aleksandra Medvedeva 14,

18000 Niš, Republic of Serbia

E-mail: nemanja.zdravkovic@elfak.ni.ac.rs 
error rates or outage probability [2]. In addition to AF and DF protocols, hybrid protocols, like the decode-amplify-forward recently arose in literature [5], which combines the benefits of both $\mathrm{AF}$ and $\mathrm{DF}$.

In the past decade, extensive literature on the topic of cooperative communications can be found [1-13]. Laneman et al. gave the mathematical framework for energy-efficient multiple-access cooperative strategies in [1]. These strategies, based on AF and DF relaying, achieve significant diversity and outage gains when compared to a non-cooperative single link case.

Besides the repetition protocols, Ikki and Ahmed have investigated best-relay and incremental-best-relay selection schemes, respectively, in [6] and [7]. In [6], instead of using all nodes in the network as relays, only the "best" one is used for forwarding data, i.e., the relay with the highest uplink signal-to-noise ratio (SNR) to the destination. In [7], this relay is used only if the destination provides a negative acknowledgement via feedback messages. With the use of these schemes, only the source node uplink channel and the best relay uplink channel are needed.

Chatzigeorgiou et al. have introduced repetition schemes over Rayleigh fading in their works regarding DF schemes, ensuring the destination will always receive the same amount of data per source node [8, 9]. In [8], in the high SNR regime, all nodes relayed data to the destination if the channel between nodes was reliable and decoding was possible at all relays. However, if the channel between nodes was poor, cooperation was dropped and every node re-sent its own data packets to the destination. In [9], the same authors improved the repetition protocol, and only those packets which are not successfully decoded were resent. These repetition protocols were further expanded in [10], where the network was subject to Nakagami- $m$ fading, while in [11] the effects of unequal SNR and temporary node blockage were taken into account.

Outage performance of low-latency DF cooperative networks was analyzed in [12,13], where a threshold-based protocol was utilized in the communication between nodes over Rayleigh fading. The protocol was based on monitoring the channel between nodes and sending data in different time periods depending on the estimated value of the instantaneous SNR.

In this paper, the main motivation is the analysis of DF cooperative networks usually found in industrial plants, which have more open layout compared to traditional wireless networks, and consist of large machines with a presence of concrete and highly reflective materials such as metal. These networks typically require low and deterministic latency. When wireless networks are used for process control, any missing or delayed data can severely degrade the quality of control [14]. Furthermore, any potential equipment problems and failures can be avoided and replacement costs can be prevented with an early notification system [15]. In these operating regimes, outage probability (OP) is the correct performance indicator.

Taking into account the previously mentioned repetitive schemes, the threshold-based protocol and temporal correlation in the channel between nodes, closed-form analytical expressions for OP are derived when the network is subject to composite fading and the destination applies maximal ratio combining (MRC). Furthermore, the impact of multipath fading and shadowing severity parameters, network dimension, average SNR values and multipath and shadowing correlation coefficients on OP is discussed.

The rest of the paper is organized as follows. Section 2 introduces the system model, the protocol used, and the composite fading channel. In Section 3, OP analysis of the system is presented. Numerical results are given in Section 4 and concluding remarks are presented in Section 5. 


\section{SYSTEM AND CHANNEL MODEL}

This paper analyzes a wireless cooperative network consisting of $U_{i}, i=1, \ldots, M$ nodes and the destination, denoted by $D$. Nodes cooperate between each other send data to the destination. The channel between cooperating nodes is referred to as the internode channel, while the channel between a node and the destination is referred to as the uplink channel. Nodes are placed in a cluster, allowing the internode channel statistics to remain the same. The destination can detect each node individually due to transmission on orthogonal channels, avoiding interference. Channel orthogonality can be achieved by means of time, frequency or code multiple access schemes (TDMA/FDMA/CDMA). All channels are subject to short-term (multipath) and long-term fading (shadowing), as well as additive white Gaussian noise (AWGN). Fig. 1 depicts a block diagram of the wireless cooperative network.

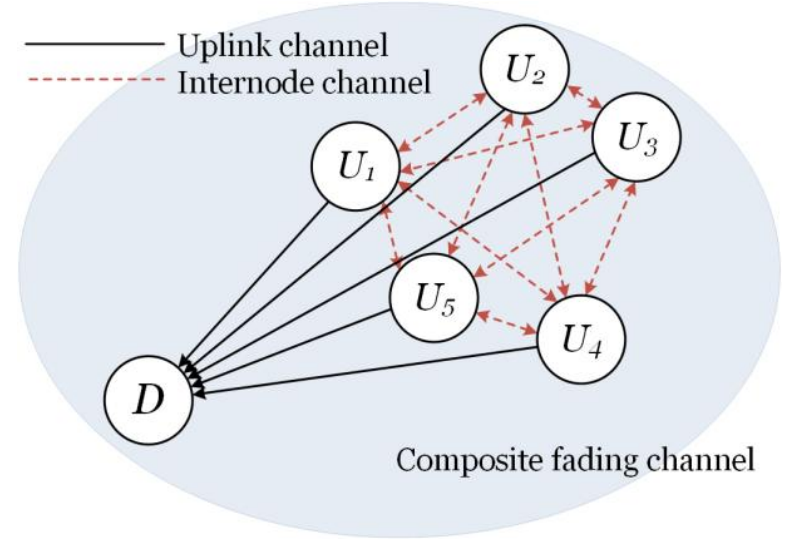

Fig. 1 Wireless cooperative network consisting of $M=5$ nodes and the destination.

The whole network is subject composite fading.

Uplink and internode channels are both subject to multipath fading and shadowing. Multipath fading, modelled by the Nakagami- $m$ distribution, has shown to be quite accurate in describing both line-of-sight as well as non-line-of-sight scenarios between the transmitting and receiving end of the communications system [16]. Fading conditions, controlled by the fading parameter $m \geq 0.5$, account both for deep fades, as well as instances where a direct signal component is present. Setting the fading parameter to $m=1$, the Nakagami- $m$ distribution reduces to the well-known Rayleigh distribution.

In addition to the multipath component, the received signal is also subject to shadowing. Empirical results showed that shadowing can be accurately described by the log-normal distribution [17]. Combining the Nakagami-m multipath component with log-normal shadowing, the unified model for composite fading will hence be Nakagami- $m$ distributed, with its mean power a random process that is log-normal $[17,18]$. However, this model is inconvenient for further mathematical manipulation and does not produce a closed-form solution. By taking an appropriate substitution for the log-normal component with the 
gamma distribution, a closed-form expression can be obtained. The resulting composite fading model is often termed in literature as the Generalized- $K\left(K_{G}\right)$ model [19].

The probability density function (PDF) and cumulative distribution function (CDF) of the uplink SNR $\gamma$ of the composite fading channel are given respectively as $[17,20]$

$$
p_{\gamma}(\gamma)=\frac{2}{\Gamma(m) \Gamma(k)}\left(\frac{m k}{\bar{\gamma}}\right)^{\frac{m+k}{2}} \gamma^{\frac{m+k}{2}-1} K_{k-m}\left(2 \sqrt{\frac{m k \gamma}{\bar{\gamma}}}\right)
$$

and

$$
P_{\gamma}\left(\gamma_{0}\right)=\frac{1}{\Gamma(m) \Gamma(k)} G_{1,3}^{2,1}\left(\frac{m k \gamma_{0}}{\bar{\gamma}} \mid \begin{array}{ccc} 
& 1 & \\
m, & k, & 0
\end{array}\right),
$$

where $m$ and $k$ are the multipath fading and shadowing severity parameters, respectively, $\bar{\gamma}$ is the average output SNR, $\Gamma(\cdot)$ is the gamma function defined in [21, eq. (1.1)], $K_{v}\left({ }^{\cdot}\right)$ is the modified Bessel function second kind of order $v$, defined in [21, eq. (8.432)] and $\mathrm{G}(\cdot)$ is the Meijer's $G$-function, defined in [21, eq. (9.301)]. In addition, Meijer's $G$-functions can be transformed in more familiar hypergeometric functions [21, eq. (9.41.1)] by applying the relation [22, eq. (07.34.26.0004.01)]. The shadowing severity parameter $k$ is related to the shadowing spread $\sigma_{S H}$ in log-normal shadowing as [17]

$$
\sigma_{S H}(\mathrm{~dB})=\frac{10}{\ln 10} \sqrt{\psi^{\prime}(k)}
$$

where $\psi^{\prime}$ is the first derivative of the digamma function, defined in [21, eq. (8.360)].

Node cooperation is performed in two subsequent stages, in the manner similar to that of [8]. During the first stage, node $U_{i}$ broadcasts its packets to all the nodes in the network and to $D$. In the second stage, if all relays have successfully decoded every packet, the destination applies MRC. Node $U_{i}$ "knows" the number of nodes that have successfully decoded its packets via feedback messages, which are assumed to be error-free. However, if any of the packets are not successfully decoded, every node drops cooperation and retransmits its own packets another $(M-1)$ times.

Each source node monitors the internode channel and applies the threshold-based transmission protocol as seen in [12,13]. At the beginning of its transmission slot, node $U_{i}$ listens to the instantaneous value of the internode SNR, denoted with $\gamma_{i}$, and compares it to the predetermined transmission threshold $\gamma_{0}$. If the SNR value of internode channel is equal or greater than $\gamma_{0}$, node $U_{i}$ sends its data packet immediately. If this is not the case, node $U_{i}$ will delay its transmission, and after a waiting period, it sends the data packet regardless of the current internode channel state. Note that even with this delay, the transmission is still within its allocated slot, causing no interference with other transmissions. However, due to the varying fading and shadowing process, the initial SNR estimate at the beginning of the transmission in the first stage, denoted with $\gamma_{i}^{\prime}$ will be correlated to the second, delayed instance $\gamma_{i}^{\prime \prime}$, both in multipath and in shadowed component. The resulting joint PDF of these two instances can be written as [19] 


$$
\begin{aligned}
p_{\gamma_{i}^{\prime}, \gamma_{i}^{\prime \prime}}\left(\gamma_{i}^{\prime}, \gamma_{i}^{\prime \prime}\right)= & \frac{4}{\Gamma(m) \Gamma(k)} \sum_{a, b=0}^{\infty} \frac{(m k)^{\xi} \rho_{n}^{a} \rho_{g}^{b}\left(\gamma_{i}^{\prime} \gamma_{i}^{\prime \prime}\right)^{\frac{\xi}{2}-1}}{\Gamma(m+a) \Gamma(k+b) \bar{\gamma}_{i}^{\xi}} \\
& \times \frac{1}{a ! b !\left(1-\rho_{n}\right)^{k+a+b}\left(1-\rho_{g}\right)^{m+a+b}} K_{\psi}\left(2 \gamma_{i}^{\prime} \sqrt{\frac{m k}{\sigma_{\gamma_{i}^{\prime}}}}\right) K_{\psi}\left(2 \gamma_{i}^{\prime \prime} \sqrt{\frac{m k}{\sigma_{\gamma_{i}^{\prime \prime}}}}\right),
\end{aligned}
$$

where $\rho_{n}$ and $\rho_{g}$ are the Nakagami- $m$ and gamma correlation coefficients, respectively, $\xi=k+m+a+b, \psi=k+b-m-a$, and $\sigma_{\gamma_{i}}=\left(1-\rho_{n}\right)\left(1-\rho_{g}\right) \bar{\gamma}_{i}$. Packet scheduling in internode communication is shown in Fig. 2.

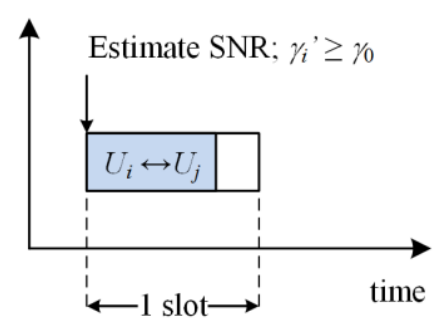

(b)

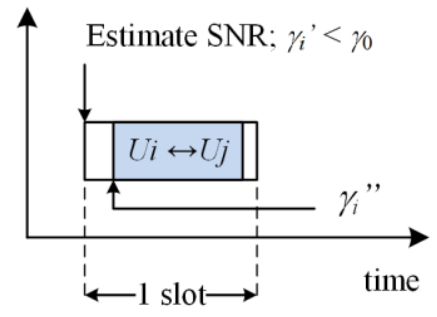

(b)

Fig. 2 Packet scheduling in the internode communication.

At the relay, a packet is successfully decoded if the internode SNR is greater than the decoding threshold $\gamma_{0}$. Mathematically speaking, the probability of successful decoding is obtained by taking a double integral over (4) as

$$
P_{\mathrm{dec}}=\int_{\gamma_{0}}^{\infty} \int_{\gamma_{0}}^{\infty} p_{\gamma_{i}^{\prime}, \gamma_{i}^{\prime \prime}}\left(\gamma_{i}^{\prime}, \gamma_{i}^{\prime \prime}\right) \mathrm{d} \gamma_{i}^{\prime} \mathrm{d} \gamma_{i}^{\prime \prime}
$$

Transforming the Bessel function in (4) to the Meijer's $G$-function from [22, eq. (03.04.26.0006.01)] and using [22, eq. (07.34.21.0084.01)] the closed-form solution for the successful decoding probability of one packet at the relay is obtained as

$$
\begin{aligned}
P_{\mathrm{dec}} & =1-\frac{1}{\Gamma(m) \Gamma(k)} \sum_{a, b=0}^{\infty} \frac{(m k)^{\xi} \rho_{n}^{a} \rho_{g}^{b} \gamma_{0}^{\xi}}{\Gamma(m+a) \Gamma(k+b) \bar{\gamma}_{i}^{\xi}} \\
& \times \frac{1}{a ! b !\left(1-\rho_{n}\right)^{k+a+b}\left(1-\rho_{g}\right)^{m+a+b}} \times\left(G_{1,3}^{2,1}\left(\frac{m k \gamma_{0}}{\sigma_{\gamma_{i}}} \mid \begin{array}{ccc}
\frac{\psi}{2}, & -\frac{\psi}{2}, & -\frac{\xi}{2}
\end{array}\right)\right)^{2} .
\end{aligned}
$$

Although (6) contains an infinite sum, this sum converges quickly. Table 1 shows the number of summed elements in (6), denoted with $N$, which are required to achieve convergence to the 6th significant digit. 
Table 1 Number of elements required for convergence to the 6th significant digit in (6)

\begin{tabular}{lcccc}
\hline$m=1, M=2$ & $\rho_{n}=0.1$ & $\rho_{n}=0.3$ & $\rho_{n}=0.5$ & $\rho_{n}=0.7$ \\
$\sigma_{S H}=3 \mathrm{~dB}$ & $\rho_{g}=0.1$ & $\rho_{g}=0.3$ & $\rho_{g}=0.5$ & $\rho_{g}=0.7$ \\
\hline $\bar{\gamma}_{i}=0 \mathrm{~dB}$ & 6 & 12 & 20 & 40 \\
$\bar{\gamma}_{i}=5 \mathrm{~dB}$ & 5 & 11 & 18 & 38 \\
$\bar{\gamma}_{i}=10 \mathrm{~dB}$ & 4 & 9 & 15 & 35 \\
$\bar{\gamma}_{i}=15 \mathrm{~dB}$ & 4 & 7 & 12 & 29 \\
\hline
\end{tabular}

To examine the convergence of infinite series in eq. (6), let's denote the finite series in eq. (6) as

$$
\begin{aligned}
& P_{\operatorname{dec}_{\mathrm{N}}}=1-\frac{1}{\Gamma(m) \Gamma(k)} \sum_{a, b=0}^{N} \frac{(m k)^{\xi} \rho_{n}^{a} \rho_{g}^{b} \gamma_{0}^{\xi}}{\Gamma(m+a) \Gamma(k+b) \bar{\gamma}_{i}^{\xi}}
\end{aligned}
$$

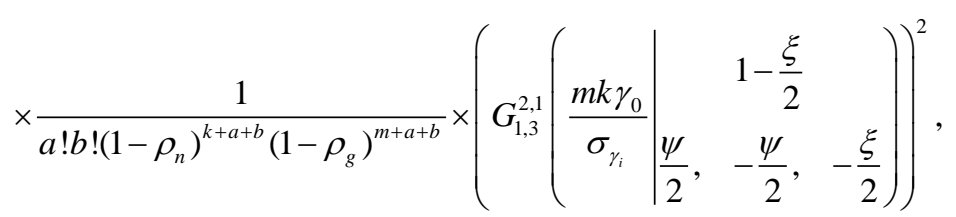

where $a$ and $b$ go from zero to $N$. Now, let's define the corresponding relative error with

$$
E_{N}=\frac{p_{\mathrm{dec}_{N}}-p_{\mathrm{dec}}}{p_{\mathrm{dec}}}
$$

One can notice that the all the terms in the series are non-negative, and $E_{N} \geq E_{N+1} \geq 0$. Through numerical evaluation of (6), satisfactory convergence is achieved, i.e., $P_{\mathrm{dec} N} \rightarrow P_{\mathrm{dec}}$ as $N \rightarrow \infty$. The convergence speed is illustrated through the behavior of $E_{N}-E_{N+1}$ for sufficiently large $N$, with the assumption $P_{\mathrm{dec}} \approx P_{\operatorname{dec}_{N+1}}$. The relative error difference can therefore be expressed as [23]

$$
E_{N}-E_{N+1}=\frac{p_{\operatorname{dec}_{N+1}}-p_{\operatorname{dec}_{N}}}{p_{\mathrm{dec}}} \approx \frac{p_{\mathrm{dec}_{N}}-p_{\mathrm{dec}_{N+1}}}{p_{\operatorname{dec}_{N+1}}} .
$$

Table 2 illustrates (9) for the parameters $m=1, \sigma_{S H}=3 \mathrm{~dB}$ and $M=2$.

Table 2 Relative error $E_{N}-E_{N+1}$

\begin{tabular}{lcccc}
\hline & $\bar{\gamma}_{i}=10 \mathrm{~dB}$ & $\bar{\gamma}_{i}=10 \mathrm{~dB}$ & $\bar{\gamma}_{i}=10 \mathrm{~dB}$ & $\bar{\gamma}_{i}=10 \mathrm{~dB}$ \\
$N$ & $\rho_{n}=0.1$ & $\rho_{n}=0.3$ & $\rho_{n}=0.6$ & $\rho_{n}=0.9$ \\
& $\rho_{g}=0.1$ & $\rho_{g}=0.3$ & $\rho_{g}=0.6$ & $\rho_{g}=0.9$ \\
\hline 15 & 0 & $1.06 \times 10^{-10}$ & $8.53 \times 10^{-6}$ & 0.0044 \\
16 & 0 & $3.11 \times 10^{-11}$ & $5.02 \times 10^{-6}$ & 0.0040 \\
17 & 0 & $9.15 \times 10^{-12}$ & $2.96 \times 10^{-6}$ & 0.0036 \\
18 & 0 & $2.69 \times 10^{-12}$ & $1.74 \times 10^{-6}$ & 0.0033 \\
19 & 0 & $7.92 \times 10^{-13}$ & $1.03 \times 10^{-6}$ & 0.0030 \\
20 & 0 & $2.33 \times 10^{-13}$ & $6.08 \times 10^{-7}$ & 0.0027 \\
\hline
\end{tabular}




\section{OUtAge Probability ANALYSiS}

After the second stage of cooperation, all nodes will choose to either forward the packet received from other nodes to the destination, or re-send their own packets instead. This decision is based on the feedback messages in the internode channels. Since every node sends a total of $M$ data packets after the end of the two stage transmission process, constant frame energy is preserved. The destination combined the received packets after the two-stage transmission process by applying MRC.

In [24], it has been shown that for identically distributed $K_{G}$ random variables, the output SNR of a MRC combined signal will remain $K_{G}$ distributed, replacing $m$ by $m M$ in the expressions for PDF and CDF everywhere except in the Meijer's $G$-function argument. In full cooperation mode, denoted with "coop", the OP can be expressed straightforward from the CDF given in (2), replacing $m$ with $m M$, obtaining

$$
P_{O_{\text {coop }}}\left(\bar{\gamma} ; \gamma_{0}\right)=\frac{1}{\Gamma(M m) \Gamma(k)} G_{1,3}^{2,1}\left(\frac{m k \gamma_{0}}{\bar{\gamma}} \mid \begin{array}{c}
1 \\
M m, k, 0
\end{array}\right) .
$$

Similarly, when cooperation is dropped and nodes act selfishly, resending their own packets, denoted with "self", there exists only one independent transmission path, but since a node sends its data packet a total of $M$ times, those transmissions are viewed as a single transmission with $M$ times the power [8,9]. The resulting expression for OP has the form

$$
P_{O_{\text {seff }}}\left(\bar{\gamma} ; \gamma_{0}\right)=\frac{1}{\Gamma(m) \Gamma(k)} G_{1,3}^{2,1}\left(\left.\frac{m k \gamma_{0}}{M \bar{\gamma}}\right|_{m, k, 0} ^{1}\right) .
$$

The network can be either in "coop" or in "self" regimes depending on the successful decoding of all packets. Due to mutual independence of the internode channels, the probability of operating on the "coop" regime is given as [8]

$$
\operatorname{Pr}[\text { coop }]=P_{\mathrm{dec}}^{M(M-1)},
$$

where $\operatorname{Pr}[\cdot]$ denotes probability and $P_{\text {dec }}$ is evaluated in (6). Similarly, the probability of being in the "self" regime is simply given as

$$
\operatorname{Pr}[\text { self }]=1-\operatorname{Pr}[\text { coop }]=1-P_{\mathrm{dec}}{ }^{M(M-1)} .
$$

Weighting the OP expressions in the "coop" and "self" regimes, given in (10) and (11) with their respectful probabilities, the final expression for OP is obtained as

$$
P_{O}\left(\bar{\gamma} ; \gamma_{0}\right)=\operatorname{Pr}[\text { coop }] P_{O_{\text {coop }}}\left(\bar{\gamma} ; \gamma_{0}\right)+\operatorname{Pr}[\text { self }] P_{O_{\text {self }}}\left(\bar{\gamma} ; \gamma_{0}\right)
$$

\section{NUMERICAL RESULTS}

In this section, numerical values for outage probability are efficiently evaluated for any given value of average SNR over internode and uplink channels, as well as fading and shadowing severity and correlation coefficients, and number of nodes in the network. The decoding and outage threshold are set to $\gamma_{0}=-0.441 \mathrm{~dB}$, which accounts for convolutional coded data packets, length of 512 bits [9]. 


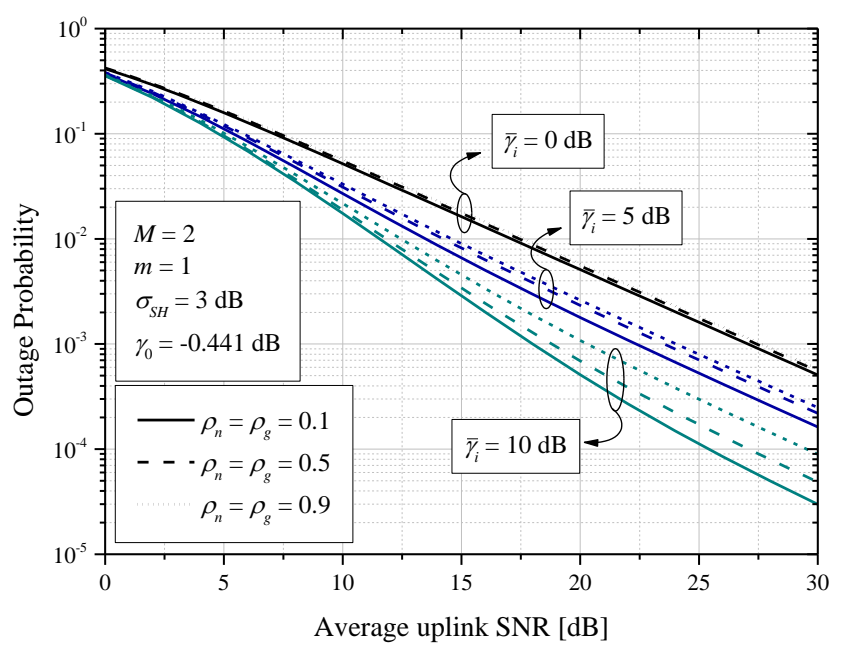

Fig. 3 Outage probability dependence on average uplink SNR for different values of average internode SNR and different correlation coefficients.

Fig. 3 shows outage probability dependence on average uplink SNR for different values of average internode SNR and different correlation coefficient combinations. As expected, increasing the average internode SNR results in lower outage probability, especially for greater values of average uplink SNR. As the internode channels improve, in the high uplink SNR regime, correlation will have a greater impact. For instance, at $\bar{\gamma}=20 \mathrm{~dB}$, outage probability drops from $1.1 \times 10^{-3}$ to $5 \times 10^{-4}$ as $\rho_{n}$ and $\rho_{g}$ decrease from 0.9 to 0.1 at $\bar{\gamma}_{i}=10 \mathrm{~dB}$, and only from $2.6 \times 10^{-3}$ to $1.7 \times 10^{-3}$ at $\bar{\gamma}_{i}=5 \mathrm{~dB}$.

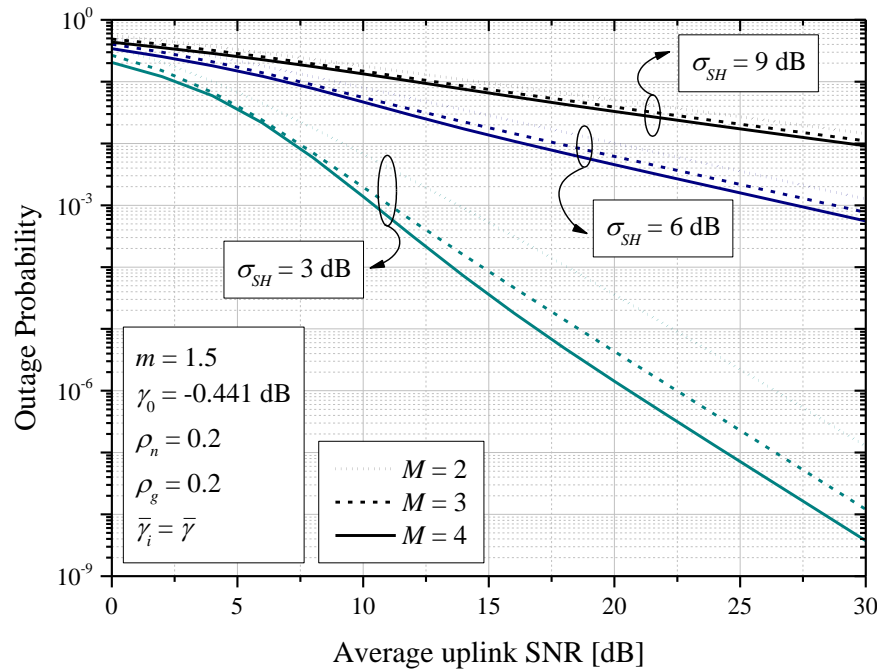

Fig. 4 Outage probability dependence on average uplink SNR

for different network dimension and different shadowing spread. 
The impact of shadowing severity is shown in Fig. 4, observed for different number of nodes in the network, for the case when average internode SNR increases simultaneously with average uplink SNR. By increasing the number of nodes in the network, we observe only a power gain, which is more noticeable for milder shadowing conditions. However, as shadowing conditions worsen, increasing the number of nodes does not significantly improve outage performance. In heavy shadowing, i.e., $\sigma_{S H}=9 \mathrm{~dB}$, even by increasing the number of nodes from $M=2$ to $M=4$, OP stays below $10^{-3}$ at $\bar{\gamma}=25 \mathrm{~dB}$. Conversely, in light shadowing conditions, at $\sigma_{S H}=3 \mathrm{~dB}$, at $\bar{\gamma}=25 \mathrm{~dB}$ the OP drops more than an order of magnitude by increasing the number of nodes from two to four.

An outage floor is noticeable when plotting outage dependence on average internode SNR in Fig. 5. When internode channels become fully reliable and data packet decoding is always possible at the relays, outage probability is saturated. By further increasing the average internode SNR no gain is observable. The value of this floor is dependent on fading and shadowing conditions, as well as average uplink SNR. For instance, for $m=1.5$ and $\bar{\gamma}=10 \mathrm{~dB}$, an outage floor of $1.3 \times 10^{-3}$ is reached at $\bar{\gamma}_{i}=17.5 \mathrm{~dB}$, while at $\bar{\gamma}=20 \mathrm{~dB}$, a floor of $4.34 \times 10^{-6}$ is reached at $\bar{\gamma}_{i}=22.4 \mathrm{~dB}$.

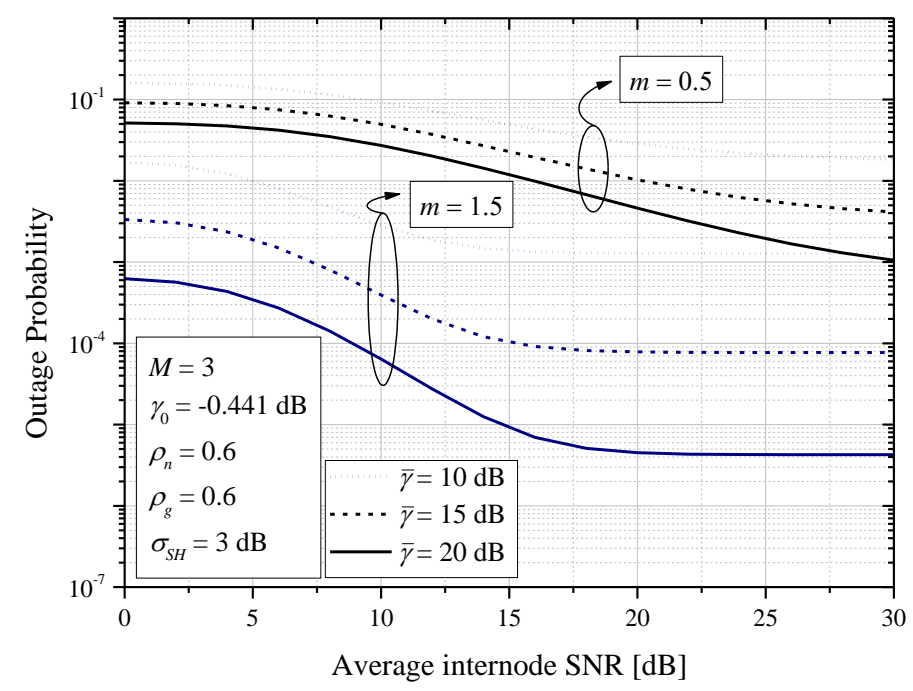

Fig. 5 Outage probability dependence on average internode SNR for different average uplink SNR and fading severity.

Finally, Fig. 6 shows outage probability dependence on multipath and shadowing correlation coefficients. As expected, better performance in obtained when either of the correlation coefficients decreases. This means that the initial and delayed internode transmissions are statistically more independent. However, the shadowing component of correlation will have less impact on outage performance than the multipath component of correlation, which can be observed by the slopes in the figure. Namely, OP decreases from more than on order of magnitude when $\rho_{n}$ deceases from one to zero, which is not the case for the shadowing correlation component, $\rho_{g}$. 


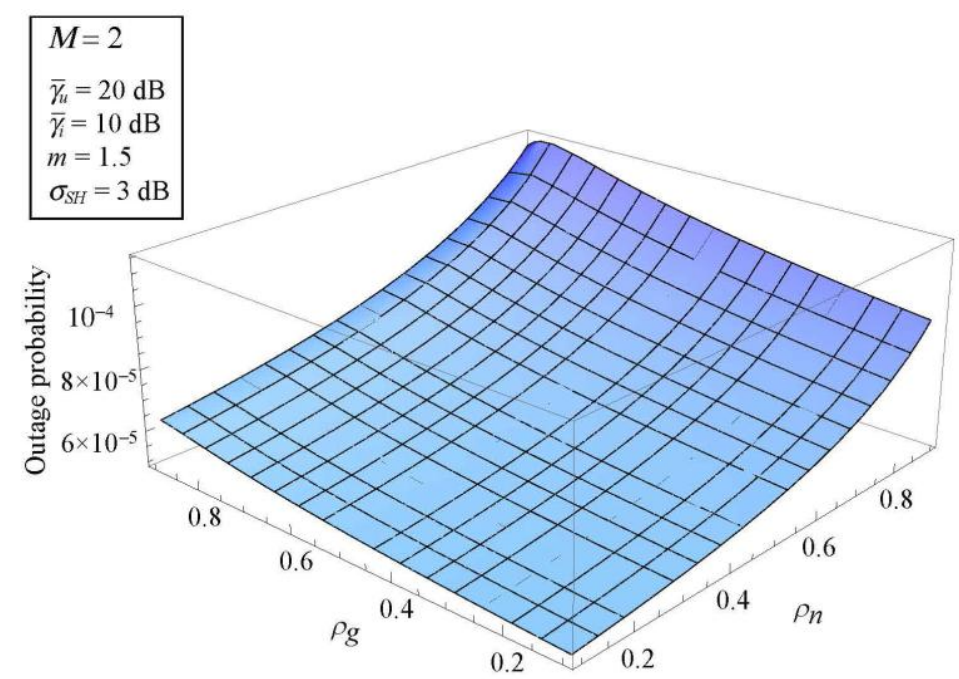

Fig. 6 Outage probability dependence on multipath and shadowing correlation coefficients.

\section{CONCLUSION}

In this paper, outage performance of a DF cooperative wireless network over a temporally correlated composite fading channel has been investigated. Based on the derived analytical expression, the outage probability dependence on different network and channel parameters, such as are fading and shadowing severity, network dimension, average SNR over uplink and internode channels, as well as fading correlation, has been analyzed.

The results have shown that the diversity gain of the network is primarily affected by the shadowing component. Higher diversity gains have been obtained in lighter shadowing conditions, while increasing the number of nodes in the network resulted in only a power gain. As internode channel conditions improved, outage performance increased until reaching an irreversible outage floor, which accounted for the case when successful packet decoding is always possible at the relays. In this regime, the impact of multipath and shadowing correlation has shown to be significant, with the multipath component being the dominant one.

Acknowledgement: This paper was supported by the Norwegian Ministry for Foreign Affairs under the project "Norwegian, Bosnian and Serbian cooperation platform for university and industry ICT $R \& D$ " NORBAS

\section{REFERENCES}

[1] J. N. Laneman, D. N. C. Tse, G. W. Wornell, "Cooperative diversity in wireless networks: efficient protocols and outage behaviour," IEEE Transactions on Information Theory, vol. 50, no. 12, pp. 30623080, 2004. [Online]. Available: http://dx.doi.org/10.1109/TIT.2004.838089

[2] M. Dohler, Y. Li, Cooperative Communications: hardware, channel \& phy, John Wiley \& Sons, United Kingdom, 2010. 
[3] A. Sendonaris, E. Erkip, B. Aazhang, "User cooperation diversity - part I: system description," IEEE Transactions on Communications, vol. 51, no. 11, pp. 1927-1938, 2003. [Online]. Available: http://dx.doi.org/ 10.1109/TCOMM.2003.818096

[4] A. Sendonaris, E. Erkip, B. Aazhang, "User cooperation diversity - part II: implementation aspects and performance analysis," IEEE Transactions on Communications, vol. 51, no. 11, pp. 1939-1948, 2003. [Online]. Available: http://dx.doi.org/10.1109/TCOMM.2003.819238

[5] X. Bao and J. Li, "Decode-amplify-forward (DAF): a new class of forwarding strategy for wireless relay channels," in Proceedings of the 6th IEEE Workshop on Signal Processing Advances in Wireless Communications (SPAWC), New York, NY, USA, 2005, pp. 816-820. [Online]. Available: http://dx.doi.org/ 10.1109/SPAWC.2005.1506253

[6] S. S. Ikki and M. H. Ahmed, "Performance analysis of adaptive decode-and-forward cooperative diversity networks with best-relay selection," IEEE Transactions on Communications, vol. 58, no. 1, pp. 68-72, Jan. 2010. [Online]. Available: http://dx.doi.org/10.1109/TCOMM.2010.01.080080

[7] S. S. Ikki and M. H. Ahmed, "Performance analysis of cooperative diversity with incremental-best-relay technique over Rayleigh fading channels," IEEE Transactions on Communications, vol. 59, no. 8, pp. 2152-2161, 2011. [Online]. Available: http://dx.doi.org/10.1109/TCOMM.2011.053111.080672

[8] I. Chatzigeorgiou, W. Guo, I. J.Wassell, and R. Carrasco, "Packet error probability for decode-andforward cooperative networks of selfish users," in Proceedings of the 10th International Symposium on Communication Theory and Applications (ISCTA), Ambleside, UK, 2009. [Online]. Available: http://www.cl.cam.ac.uk/research/dtg/www/publications/public/ic231/ic231_ISCTA09_camready.pdf

[9] I. Chatzigeorgiou, W. Guo, I. J.Wassell, and R. Carrasco, "Exact and asymptotic outage probability analysis for decode-and-forward networks," IEEE Transactions on Communications, vol. 59, no. 2, pp. 376-381, 2011. [Online]. Available: http://dx.doi.org/10.1109/TCOMM.2010.102910.090133

[10] A. Cvetkovic, K. Kansanen, and G. T. Djordjevic, "Outage analysis for uncoordinated decode-andforward networks over Nakagami-m channels," in Proceedings of the 20th Telecommunications Forum (TELFOR), Belgrade, Serbia, 2012, pp. 490-493. [Online]. Available: http://dx.doi.org/10.1109/TELFOR. 2012.6419254

[11] G. T. Djordjevic, K. Kansanen, and A. Cvetkovic, "Outage performance of decode-and-forward cooperative networks over Nakagami- $m$ fading with node blockage," IEEE Transactions on Wireless Communications, vol. 15, no. 9, pp. 5848-5860, 2016. [Online]. Available: http://dx.doi.org/10.1109/ TWC.2016.2572082

[12] N. Zdravkovic, A. Cvetkovic, G. T. Djordjevic, and K. Kansanen, "Outage probability of decode-andforward network with threshold based protocol over Rayleigh fading," in Proceedings of the 21st Telecommunications Forum (TELFOR), Belgrade, Serbia, 2013, pp. 315-318. [Online]. Available: http://dx.doi.org/10.1109/TELFOR.2013.6716233

[13] N. Zdravkovic, A. Cvetkovic, K. Kansanen, and G. T. Djordjevic, "Outage performance of low-latency decode-and-forward cooperative wireless networks," EURASIP Journal on Wireless Communications and Networking, vol. 2016, no. 1, pp. 1-10, 2016. [Online]. Available: http://dx.doi.org/10.1186/s13638-016-0592-6

[14] S. Han, X. Zhu, A. K. Mok, D. Chen, and M. Nixon, "Reliable and real-time communication in industrial wireless mesh networks," in Proceedings of the 17th IEEE Real-Time and Embedded Technology and Applications Symposium (RTAS), Chicago, IL, USA, 2011, pp. 3-12. [Online]. Available: http://dx.doi.org/ 10.1109/RTAS.2011.9

[15] E. Tanghe, W. Joseph, L. Verloock, L. Martens, H. Capoen, K. V. Herwegen, and W. Vantomme, "The industrial indoor channel: large-scale and temporal fading at 900, 2400, and $5200 \mathrm{MHz}$, "IEEE Transactions on Wireless Communications, vol. 7, no. 7, pp. 2740-2751, 2008. [Online]. Available: http://dx.doi.org/10.1109/TWC.2008.070143

[16] M. K. Simon and M.-S. Alouini, Digital communication over fading channels, 2nd ed. John Wiley \& Sons, USA, 2005.

[17] P. Shankar, "Error rates in generalized shadowed fading channels," Wireless Personal Communications, vol. 28, no. 3, pp. 233-238, 2004. [Online]. Available: http://dx.doi.org/10.1023/B:wire.0000032253. 68423.86

[18] I. M. Kostic, "Analytical approach to performance analysis for channel subject to shadowing and fading," IEE Proceedings - Communications, vol. 152, no. 6, pp. 821-827, 2005. [Online]. Available: http://dx.doi.org/10.1049/ip-com:20045126

[19] P. S. Bithas, N. C. Sagias, P. T. Mathiopoulos, G. K. Karagiannidis, and A. A. Rontogiannis, "On the performance analysis of digital communications over generalized-k fading channels," IEEE Communications 
Letters, vol. 10, no. 5, pp. 353-355, 2006. [Online]. Available: http://dx.doi.org/10.1109/LCOMM. 2006.1633320

[20] N. D. Chatzidiamantis and G. K. Karagiannidis, "On the distribution of the sum of gamma-gamma variates and applications in RF and optical wireless communications," IEEE Transactions on Communications, vol. 59, no. 5, pp. 1298-1308, 2011. [Online]. Available: http://dx.doi.org/10.1109/ TCOMM.2011.020811.090205

[21] I. S. Gradshteyn, I. M. Ryzhik, Table of Integrals, Series, and Products. 5th ed., New York: Academic, 1994, USA.

[22] The wolfarm functions site. [Online]. Available: http://functions.wolfram.com/ [Accessed on December 2016]

[23] G. V. Milovanovic, M. C. Stefanovic, S. R. Panic, J. A. Anastasov, and D. S. Krstic, "Statistical analysis of the square ratio of two multivariate exponentially correlated $\alpha-\mu$ distributions and its application in telecommunications," Mathematical and Computer Modelling., vol. 54, no. 12, pp. 152- 159, 2011. [Online]. Available: http://dx.doi.org/10.1016/j.mcm.2011.01.046

[24] P. M. Shankar, "Outage probabilities of a MIMO scheme in shadowed fading channels with micro- and macrodiversity reception," IEEE Transactions on Wireless Communications, vol. 7, no. 6, pp. 2015-2019, 2008. [Online]. Available: http://dx.doi.org/10.1109/TWC.2008.070053 